\title{
Envolvimento de micoplasmas em vacas com distúrbios reprodutivos
}

\author{
Mycoplasmas involvement in cows with reproductive disorders \\ Maria da Graça Fichel do Nascimento' ${ }^{1}$, Flora Helena de Freitas D'Angelis² \\ Elmiro Rosendo do Nascimento ${ }^{3}$ \& Osvaldo de Almeida Resende ${ }^{4}$
}

\begin{abstract}
RESUMO
Microrganismos pertencentes ao gênero Mycoplasma têm sido isolados do trato genital e de sêmem bovino, podendo expressar patogenicidade ou não. O objetivo deste estudo foi verificar a presença de Mycoplasma spp., e comparar com o diagnóstico de três importantes doenças da reprodução, em 14 vacas que apresentavam distúrbios reprodutivos. Foram coletadas amostras de muco vaginal de cada animal, usando-se solução salina tamponada a $0,85 \%$ para as coletas, transferindo-se alíquotas simultaneamente para o meio de Hayflick modificado, para isolamento de Mycoplasma spp., e para o meio de Lactopep para diagnóstico da Tricomonose bovina. Também foram coletadas amostras para pesquisa de anticorpos: de muco vaginal em tampão de gaze para a prova de muco aglutinação lenta, para o diagnóstico de Campilobacteriose e amostras de sangue para o teste de soroaglutinação em placa, para o diagnóstico de Brucelose. Das 14 amostras examinadas, oito $(57,1 \%)$ foram positivas para Mycoplasma spp., e todas (100\%) foram negativas para Brucelose, Tricomonose e Campilobacteriose, principais doenças da reprodução, registrando-se a ocorrência de microrganismos do gênero Mycoplasma no trato genital de vacas com problemas reprodutivos, sugerindo-se seus envolvimentos como patógenos.
\end{abstract}

Descritores: Mycoplasma, bovino, trato genital, infertilidade.

\section{ABSTRACT}

Microorganisms of the genus Mycoplasma have been isolated from the genital tract and from semen of bovine, and they can be pathogenic or not. The objective of this study was to verify the presence of Mycoplasma spp., and to compare with the diagnosis of other three important reproductive diseases, in 14 cows with history of reproductive disorders. Vaginal mucus samples were collected from each animal by using buffer saline solution at $0.85 \%$, followed by the transferring of aliquots, simultaneously, to the modified Hayflick medium, for Mycoplasma isolation, and to Lactopep medium, for the diagnosis of Bovine Tricomoniasis. Additionally, samples were also collected for immunodiagnosis: from vaginal mucus, collected in a gauze buffer, for the lent mucus agglutination test, for the diagnosis of Campylobacteriosis, and blood samples for the standard plate agglutination test, for the diagnosis of Brucellosis. From the 14 samples examined, eight (57.1\%) were positive for Mycoplasma spp., and all of them (100\%) were negative to Brucellosis, Tricomoniasis and Campilobacteriosis. These results acknowledge the occurrence of microorganisms of the genus of Mycoplasma in the genital tract of cows with reproductive problems, in Brazil, suggesting their pathogenic involvement.

Key words: Mycoplasma, bovine, genital tract, infertility. 


\section{INTRODUÇÃO}

As doenças genitais em bovinos contribuem sensivelmente para a diminuição da produção de leite e carne, sendo ainda os agentes infecciosos responsáveis por sérios problemas na reprodução, produzindo abortos e, consequientemente, infertilidade. Microrganismos pertencentes ao gênero Mycoplasma são apontados como responsáveis por diversas patogenias genitais de bovinos, podendo levar a repetição de cio por impedimento da fertilização ou interferência no desenvolvimento inicial do embrião [5,10,14,18,21-23]. A transmissão venérea pode ocorrer principalmente nos casos em que $M$. bovis, $M$. canadense e M. bovigenitalium estejam envolvidos [6,18,20,21]. Além disso, vulvovaginite granular já foi produzida em novilhas livres de doenças através de inoculação experimental de $M$. bovigenitalium, comprovando sua patogenicidade nos distúrbios reprodutivos [22].

Embora existam alguns relatos de doenças reprodutivas bovinas causadas experimentalmente por certas espécies de Mycoplasma além de vários relatos de isolamento, em outros países [1,3,5-8,11,18-20,22, 23,25,27], no Brasil existem poucos trabalhos sobre o envolvimento de micoplasmas em trato reprodutivo bovino [4,14-17,24]. Nascimento et al. [16] isolaram pela primeira vez no Brasil, cepas de M. bovirhinis, de muco vaginal de novilhas com vulvovaginite granular. Adicionalmente, também foram isolados micoplasmas de touros procedentes de rebanho com problemas reprodutivos [15].

Neste trabalho, verificou-se a presença de organismos do gênero Mycoplasma no trato genital de vacas com problemas reprodutivos, adaptando-se para seu

Tabela 1. Positividade para Mycoplasma spp. em amostras de muco vaginal de 14 fêmeas bovinas, por tipo de distúrbio reprodutivo, de uma propriedade localizada no Estado de Minas Gerais.

\begin{tabular}{|c|c|c|c|}
\hline \multirow{2}{*}{$\begin{array}{c}\text { Distúrbio } \\
\text { Reprodutivo }\end{array}$} & \multicolumn{2}{|c|}{ Fêmeas Diagnosticadas ${ }^{\star *}$} & \multirow{2}{*}{ Total de Fêmeas } \\
\hline & Positivas $\mathbf{N}^{\circ}(\%)$ & Negativas $\mathbf{N}^{\circ}(\%)$ & \\
\hline Ausente & $1(7,14)$ & $1(7,14)$ & 2 \\
\hline Natimorto & $3(21,43)$ & $2(14,29)$ & 5 \\
\hline Aborto & $2(14,29)$ & $3(21,43)$ & 5 \\
\hline VVD/Aborto* & $1(7,14)$ & $0(0,00)$ & 1 \\
\hline VVD & $1(7,14)$ & $0(0,00)$ & 1 \\
\hline Total & $8(57,14)$ & $6(42,86)$ & 14 \\
\hline
\end{tabular}

diagnóstico um método de coleta usado para Tricomonose, e comparou-se (ou se estabeleceu comparação) com os diagnósticos de Tricomonose, Campilobacteriose e Brucelose.

\section{MATERIAIS E MÉTODOS}

Amostras de muco vaginal foram coletadas de 14 vacas mestiças de Charolês com Nelore. Os animais apresentavam diversos sinais clínicos de patologias reprodutivas (Tabela 1).

Para a coleta de material, adaptou-se o uso de solução salina tamponada a $0,85 \%$, transferindo-se alíquotas simultaneamente para o isolamento de $\mathrm{Myco-}$ plasma spp., para meio líquido de Hayflick [20], usado para transporte e cultivo, e para o meio de Lactopep, conforme preconizado, para diagnóstico de Tricomonose bovina, segundo técnica empregada por Mello \& Guida (1954), apud Lopes [12].

Amostras de muco vaginal foram também coletadas para diagnóstico de Campilobacteriose, pelo método de mucoaglutinação lenta [9] além de amostras de sangue para exames de Brucelose, pelo método da soroaglutinação rápida em placa, segundo normas do Ministério da Agricultura [2]. Com exceção das amostras de sangue, as demais amostras coletadas foram acondicionadas em caixa térmica com gêlo, até a chegada ao laboratório, onde as amostras de muco vaginal, acondicionadas em meio líquido de Hayflick, foram inoculadas em meios sólido (placas) e líquido (tubos com tampa) de Hayflick.

Os cultivos em placa foram incubados em microaerofilia, a $37^{\circ} \mathrm{C}$, utilizando-se jarra com vela acesa, até aproximadamente 10 dias, sendo examinadas a cada dois dias, em microscópio estereoscópico, para visualização de colônias em forma de "ovo frito". Todos os cultivos seguiram os procedimentos adotados para isolamento de micoplasmas [21,26].

As proporções de resultados positivos e negativos para micoplasma, por tipo de distúrbio, foram comparadas pelo teste de Qui-quadrado, com intervalo de confiança de 95\% [13].

\section{RESULTADOS}

Os resultados para os exames de Brucelose, Campilobacteriose e Tricomonose foram todos negativos, o que exclui 
a possibilidade de uma dessas enfermidades ter sido responsável pelos distúrbios reprodutivos que os animais apresentavam (Tabela 1). Entretanto, das 14 amostras de muco vaginal, $57,15 \%$ (8/14) foram positivas para o isolamento de Mycoplasma spp., sendo somente $7,15 \%$ (1/4) sem distúrbios reprodutivos. Inicialmente, os micoplasmas caracterizaram-se pela formação de colônias típicas em forma de "ovo frito" e confirmadas posteriormente pela coloração de Dienes, sendo uma delas classificada como M. bovis (Tabela 1). Quatro das 14 cepas não fermentaram glicose, mas hidrolizaram arginina, caracterizando a existência de mais de uma espécie de Mycoplasma.

\section{DISCUSSÃO}

A taxa de ocorrência de micoplasma de 57,15\%, obtida nesse estudo, foi muito superior àquelas descritas em outros países $[3,11]$ representadas, respectivamente, por $11,0 \%$ e $21,0 \%$, o que nos leva a crer que o método de coleta empregado é bastante promissor.

Das cinco vacas que abortaram, duas foram positivas para o isolamento do Mycoplasma spp. (Tabela 1), o que sugere o possível envolvimento desta bactéria em aborto bovino. Dados semelhantes foram relatados anteriormente de genitália de fêmeas bovinas, bem como de fetos abortados e de placenta [11,25]. Podese observar que os dois animais com vulvovaginite discreta foram positivos para micoplasma (Tabela 1), o que reforça a hipótese do possível envolvimento de $\mathrm{Myco-}$ plasma nas patologias reprodutivas bovina. Adicionalmente, embora não fosse o objetivo deste estudo, classificou-se um dos isolados, amostra obtida de vaca com vulvovaginite e que havia abortado, como Mycoplasma bovis (M.bovis), cuja patogenicidade genital e/ou reprodutiva já é bastante conhecida $[11,25]$.

Pelo teste de Qui-quadrado, houve diferenças estatisticamente significativas $(p<0,0045)$ entre as proporções de resultados positivos e negativos, por tipo de distúrbio reprodutivo, sendo a maior diferença para VVD e VVD/Aborto.

De acordo com os resultados desse estudo (Tabela 1), pôde-se verificar adicionalmente, que a técnica utilizada para a coleta de amostras de muco vaginal em solução salina tamponada, adaptada para o diagnóstico de isolamento de Mycoplasma, foi eficiente, podendo portanto, ser recomendada para o isolamento desse microrganismo de fêmeas bovinas.
Sabe-se que a presença de microrganismos patogênicos introduzidos pela transferência de embriões, ou outros métodos (inseminação artificial), resulta em infecções no trato reprodutivo e conseqüentemente infertilidade [8]. A presença de micoplasmas no aparelho genital de fêmeas bovinas pode repercutir em doenças genitais, e esses animais podem transmitir esse microrganismo a outros animais e causar infertilidade. A maioria dos estudos envolvendo isolamento e/ou patogenicidade de Mycoplasma spp. de trato reprodutivo bovino, por infecção natural ou experimental, foi realizada na década de 90 e nenhum deles procurou relacionar o distúrbio encontrado com a presença de outras enfermidades correlatas.

No presente trabalho, o diagnóstico positivo de Mycoplasma spp. através de isolamento, do trato genital de vacas com problemas reprodutivos e com diagnóstico negativo para Tricomonose, Campilobacteriose e Brucelose, tornou-se um fato altamente sugestivo da patogenicidade dos micoplasmas isolados. Além disso, a presença de $M$. bovis em animal com distúrbio reprodutivo confirma as suspeitas de envolvimento de $\mathrm{Myco}$ plasma e sua patogenicidade. A Micoplasmologia, por ser uma área de dedicação praticamente exclusiva, bastante complexa e demandar muito trabalho laboratorial, talvez justifique a carência de estudos semelhantes, que envolvam diagnóstico conjunto de micoplasmose e outras enfermidades reprodutivas, os quais não foram encontrados na literatura, embora mereçam ser conduzidos. Adicionalmente, o diagnóstico de Mycoplasma spp. de bovinos, por isolamento, é bem mais difícil do que o de outras espécies animais, por existir cepas cujo crescimento em meio líquido é quase imperceptível.

\section{CONCLUSÃO}

Os sinais clínicos observados, somados aos resultados positivos de isolamento de Mycoplasma spp., sendo um deles $M$. bovis, e a ausência de outros patógenos pesquisados, quer pela tentativa de isolamento ou imunodiagnóstico, são altamente sugestivos do envolvimento dos micoplasmas, em distúrbios reprodutivos em bovinos. Desta forma, recomenda-se o diagnóstico das micoplasmoses na etiolopatogenia das enfermidades reprodutivas bovinas, incluindo outras doenças não contempladas no presente estudo. 


\section{REFERÊNCIAS}

1 Afshar A., Stuart P. \& Huck R.A. 1966. Granular vulvovaginitis (Nodular Venereal Disease) of cattle associated with $M y c o-$ plasma bovigenitalium. Veterinary Record. 78: 512-519.

2 Brasil - Legislação de Defesa Sanitária Animal. 1991. Manual de procedimentos- Movimentação interestadual de animais e produtos. Ministério da Agricultura. Secretaria Nacional de Defesa Agropecuária (Portaria nº. 23/76 de janeiro de 1976). Brasília: Serviço de Defesa Sanitária Animal, pp.56-63.

3 Cottew G.S. 1970. Mycoplasma isolated from cattle in Australia. Australian Veterinary Journal. 46: 378-381.

4 D’Angelis F.H.F., Nascimento M.G.F., Resende O.A. \& Nascimento E.R. 1996. Mycoplasma bovis in cases of bovine vulvovaginitis in Brazil. [Sessão PN 13, №.033]. In: Resumos do XV Congresso Panamericano de Medicina Veterinária-PANVET (Campo Grande, Brasil). p.409.

5 Doig P.A. 1981. Bovine genital mycoplasmosis. Canadian Veterinary Journal. 22: 339-343.

6 Eaglesome M.D. \& Garcia M.M. 1990. The effect of Mycoplasma bovis on fertilization processes in vitro with bull spermatozoa and zona-free hamster oocytes. Veterinary Microbiology. 21: 329-337.

7 Friis N.F. \& Blom E. 1983. Isolation of Mycoplasma canadense from bull semen. Acta Veterinary Scandinavica. 24: $315-$ 317.

8 Gourlay R.N. \& Howard C.J. 1979. Bovine mycoplasmas. In: Tully J.G. \& Whitcomb R.F. (Eds). The Mycoplasmas. Human and Animal Mycoplasma. New York: Academic Press, v.2, pp.49-102.

9 Kendrick J.W. 1967. The vaginal mucus agglutination test for Bovine Vibriosis. Journal of the American Veterinary Medical Association. 150: 495-498.

10 Kunz T.L., Gambarini M.L., Oliveira Filho B.D. \& Galindo A.D.S. 2002. Mortalidade embrionária em bovinos: interrelações embrião-patógenos. Revista do Conselho Federal de Medicina Veterinária - Suplemento Técnico. [Online]. 26: 1-42. Disponível em: <http://www.cfmv.org.br/rev26/tecnico8.htm>. Acessado em: 08/03/2004.

11 Landford E.V. 1975. Mycoplasma species recovered from the reproductive tracts of Western Canadian cows. Canadian Journal of Comparative Medicine. 39: 133-137.

12 Lopes L.M.S. 1990. A utilização de um meio à base de peptona e leite para transporte e cultivo de Tritrichomonas foetus (RIEDMULLER, 1928). 160f. Niterói, RJ. Dissertação (Mestrado) - Programa de Pós-graduação em Medicina Veterinária, Universidade Federal Fluminense.

13 Martin S.W., Meek A.N. \& Willeberg P. 1994. Veterinary Epidemiology: Principles and Methods. Ames: Iowa State University Press, 343p.

14 Mettifogo E. 2000. Efeitos da infecção por micoplasmas no trato reprodutivo de bovinos: diagnóstico, controle e tratamento - Revisão. Revista Brasileira de Reprodução Animal. 24: 83-89.

15 Nascimento E.R., D’Angelis F.H.F., Nascimento M.G.F. \& Resende O.A. 1998. Isolamento de micoplasmas de touros provenientes de um rebanho com problemas reprodutivos. Revista Brasileira Medicina Veterinária. 20: 158-160.

16 Nascimento M.G.F., D’Angelis F.H.F., Resende O.A., Nascimento E.R. \& Lignon G.B. 1996. Isolation of Mycoplasma bovihrinis form vaginal mucus of heifers. [Sessão PN 13, №.032]. In: Resumos do XV Congresso Panamericano de Medicina Veterinária - PANVET (Campo Grande, Brasil). p.407.

17 Nascimento M.G.F., D’Angelis F.H.F., Resende O.A., Nascimento E.R. \& Lignon G.B. 1998. Mycoplasma bovirhinis em muco vaginal de novilhas. Revista Brasileira Medicina Veterinária. 20: 210-214.

18 Parsonson I.M., Al-Aubaidi J.M. \& McEntee K. 1974. Mycoplasma bovigenitalium: experimental induction of genital disease in bulls. Cornell Veterinarian. 64: 240-264.

19 Panangala V.S., Fish N.A. \& Barnum D.A. 1978. Microflora of the cervico-vaginal mucus of repeat breeder cows. Canadian Veterinary Journal. 19: 83-89.

20 Rae A.G. 1982. Isolation of Mycoplasmas from bovine semen. The Veterinary Record. 111: 462.

21 Ruhnke H.L. 1994. Mycoplasmas associated with bovine genital tract infections. In: Whifford H.W., Rosenbusch R.F. \& Lauerman L.H. (Eds). Mycoplasmosis in Animals. Laboratory Diagnosis. Ames: Iowa State University Press, pp.56-64.

22 Saed O.M. \& Al-Aubaidi J.M. 1983. Infertility in heifers caused by pathogenic strain of Mycoplasma bovigenitalium. Cornell Veterinarian. 73: 125-130.

23 Sylla L., Stradioli G., Manuali E., Rota A., Zelli R., Vicenti L. \& Monaci M. 2005. The effect of Mycoplasma mycoides ssp. Mycoides LC of bovine origen on in vitro fertilizing ability of bull spermatozoa and embryo development. Animal Reproduction Science. 85: 81-93. 
24 Terasaki M.H.F. 1990. Mycoplasma: Isolamento no sêmen bovino e caracterização. Seropédica, RJ. 105f. Dissertação (Mestrado em Medicina Veterinária Preventiva) - Programa de Pós-graduação em Medicina Veterinária, Universidade Federal Rural do Rio de Janeiro.

25 Trichard C.J.V. \& Jacobsz E.P. 1985. Mycoplasmas recovered from bovine genitalia, aborted foetuses and placentas in the Republic of South Africa. Onderstepoort Journal of Veterinary Research. 52: 105-110.

26 Tully J.G. 1983. General cultivation techniques for mycoplasmas and spiroplasmas. In: Razin S. \& Tully J. (Eds). Methods in Mycoplasmosis. Mycoplasma Characterization. 6th edn. New York: Academic Press, pp.99-101.

27 Vandeplassche M. 1982. Herd infertility: Mycoplasma abortion. In: FAO Reproductive Efficiency in Cattle: a Guideline for Projects in Developing Countries. Roma: FAO, pp.1-98. 